\title{
Die Küstenfischerei in der Unter- und Außenweser und die Abwasserbedrohung
}

\author{
WiLly Nolte \\ Staatliches Fischereiamt, Bremerbaven
}

\begin{abstract}
The inshore-fishery in the lower and outer Weser and the danger of water pollution. In earlier times the fishery of the lower and outer Weser was of a high commercial value. Todays fishery suffers from the development of the Weser River into a great shipping way and increasing water pollution. Many professional fishermen have been replaced by hobby fishermen; these fish in great numbers and, therefore, secure relatively great quantities of fish. In 1966 the value of the total catch of professional and hobby fishermen amounted to about 3 million DM. Such high yields can, however, hardly be expected in the future. Already now the Weser belongs to the $\alpha$-to $\beta$-mesosaprob zone. The greatest danger is to be seen in the trend of the various industries to settle near the coast and to dump their wastes into rivers and littoral areas expecting fast and complete mixing of the waters. However, the Weser estuary is always stratified horizontally and vertically, hence total mixing wastes never occurs. In view of the great amounts of industrial wastes to be discharged and the hydrographic stratification, severe biological consequences are to be expected. Some of the potential water pollution damages are discussed.
\end{abstract}

\section{DIE FISCHEREI IN DER UNTER- UND AUSSENWESER}

Die Fischerei in der Weser gehört nach dem Fischereigesetz von Bremen-Vegesack abwärts zur Küstenfischerei. In alten Zeiten hat sie einen hohen Wert gehabt; denn es hätten sich sonst wohl nicht Kaiser und hohe Gerichte damit befaßt. Das geht hervor aus den kaiserlichen Schenkungen des Fischereiregals an das Kloster Corvey 832, an das Erzbistum Bremen 937 und 1062 sowie schließlich 1541 auf dem Reichstag zu Regensburg durch Karl V. an die Freie Stadt Bremen "von der Brücke in Hoya bis zur salzenen See, wie solches alles über etliche hundert Jahre unverhindert hergebracht".

Später folgten viele Rechtsstreite, die bis zum Oberappellationsgericht, Kammergericht und selbst bis zum Reichsgericht gingen. Aus diesen Gründen ist hier der Fischfang nicht frei, sondern nur mit gebührenpflichtiger Erlaubnis zulässig.

Heute ist der Wert der Stromfischerei in der Unterweser stark beeinträchtigt durch die Eingriffe des Menschen in die Natur, und zwar durch: (1) Ausbau der Weser als Großschiffahrtsstraße seit 1887, der zum Beispiel den Tidenhub in Bremen von 0,30 auf 3,20 m steigen ließ; (2) zunehmende Belastung der Weser mit Abwässern der Siedlungen und der Industrie. Der größere Einfluß kam wohl bisher von dem Stromausbau. Mit dem Tidenhub wälzt sich täglich zweimal eine gegen früher gewaltig ver- 
größerte Wassermasse durch das Flußbett hinauf und hinunter. Die Strömung hat daher stark zugenommen, das betonnte Fahrwasser ist bis unmittelbar an die Buhnenköpfe und Deckwerke ausgedehnt worden, so daß technisch kaum noch Fangplätze für die Fischer verblieben sind. Die schnelle Fahrt der großen Seeschiffe verursacht einen übermäßigen Sog und Schwell, der die Hamenkutter bedroht (Abb. 1).

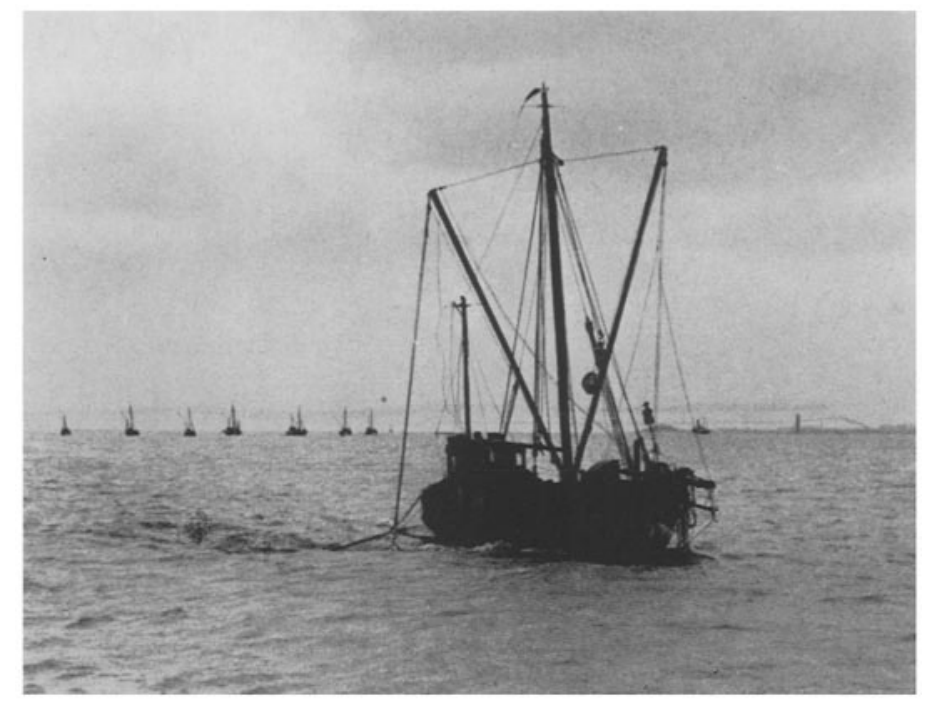

Abb. 1: Hamenkutter beim Fang vor Anker in der Wesermündung (um 1955). Im Hintergrund liegen weitere 7 Kutter. Heute haben nur noch 2 bis 3 Platz

Demgegenüber ist der Einfluß der Abwässer bisher nicht so gravierend, denn im allgemeinen wird die biologische Selbstreinigung des Stromes noch damit fertig. Allerdings gibt es im Sommer auch schon gelegentlich Fischsterben. Durch die Entwicklung ist die Weserfischerei aber im Laufe der Jahrzehnte seit 1900 erheblich verändert worden. Der Stör (Acipenser sturio L.) ist trotz aller Schonmaßnahmen - vermutlich aus biologischen Gründen - verschwunden, wie fast an der ganzen Nordseeküste. Der Lachs (Salmo salar L.) ist durch den Bau der Talsperren in den Oberläufen der Flüsse und durch Wehre von seinen Laichplätzen abgeschnitten worden (MüLLER 1960), in der Weser besonders durch die Edertalsperre (1906 bis 1910). In der Fulda gab es zum Beispiel 1443 in einem Zug 798 Lachse, 1649 noch 239 Lachse und 1848 im Frühjahr mehrere hundert in Eder und Fulda. Den letzten Schlag versetzte der Lachsfischerei dann das Wehr bei Bremen-Hemelingen 1911. Im Jahre 1890 wurden dort 10000 Lachse gefangen, 1900: 4000, 1910: 3000 und 1920 nur noch 250 Lachse.

Ahnlich erging es dem Schnepel (Coregonus oxyrbynchus L.) und dem Maifisch (Alosa finta Cuv.) (1938: $96000 \mathrm{~kg}, 1950: 38000 \mathrm{~kg}, 1960: 1000 \mathrm{~kg}$ ), ferner der Quappe (Lota lota L.) und von Brake an aufwärts den früher dort wichtigen Süßwasserfischen, darunter dem Hecht (Esox lucius L.). In alten Urkunden von 1659, 1738 und 1800 ist nachzulesen, daß dem Herkommen nach von den Bremer Stadtfischern zu 
Ostern und Martini dem Rat der Stadt und dem Erzbischof Quappen und Hechte als Deputat zu liefern waren. Heute würden sie dieser Verpflichtung nicht mehr nachkommen können.

Mit den Fangmöglichkeiten nahm auch die Zahl der Berufsfischer ab; sie belief sich 1900 auf 200, 1910 auf 150, 1925 auf 102, 1950 auf 97 und 1965 auf 20 Fischer. Trotz dieser Abnahme ist die Zahl der vom Staat gegen Gebühren für die Weser ausgegebenen Fischereikarten nicht geringer, sondern größer geworden. Nachdem um 1908 noch ein Numerus clausus die Zahl der großen Fischereikarten, und damit die Zahl der Berufsfischer, auf 150 begrenzte, werden heute diese Karten auch an Neben- und Sportfischer ausgegeben. Seit der Durchsetzung der Fünftagewoche stieg die Zahl wieder, da vielen heute die nötige Freizeit zur Verfügung steht, um der Nebenfischerei nachzugehen. Auch die Gebühr von 40,- DM schreckt niemanden ab. 1966 wurden 154 große Karten gezählt.

Tabelle 1

Fänge in der Unterweser (in kg)

\begin{tabular}{|cccc|}
\hline Jahr & Aal & Stint & DM \\
\hline 1955 & 45000 & 133000 & 159000 \\
1960 & 51000 & 96000 & 207000 \\
1966 & 71000 & 65000 & 337000 \\
\hline
\end{tabular}

Tabelle 2

Fänge in der Außenweser 1966 (in t)

\begin{tabular}{|lrccc|}
\hline \multicolumn{1}{c}{ Häfen } & Kutter & Speisekrabben & Futterkrabben & Beifänge \\
\hline Fedderwardersiel & 23 & 359 & 1318 & 60 \\
Bremerhaven & 1 & 19 & 70 & -14 \\
Wremen & 6 & 157 & 570 & 458 \\
Dorum (Abb. 3) & 18 & 445 & 1829 & 1 \\
Spieka & 8 & 243 & 967 & 533 \\
\hline$\Sigma$ & 56 & 1233 & 4754 & \\
\hline
\end{tabular}

Tabelle 3

Erlöse der Fänge in der Außenweser (in DM)

\begin{tabular}{|lrr|}
\hline \multicolumn{1}{|c}{ Häfen } & 1965 & 1966 \\
\hline Fedderwardersiel & 497000 & 748000 \\
Bremerhaven & 30000 & 40000 \\
Wremen & 242000 & 329000 \\
Dorum & 652000 & 979000 \\
Spieka & 310000 & 513000 \\
\hline$\Sigma$ & 1731000 & 2609000 \\
\hline
\end{tabular}


Die Fischerei ist fast ganz zurückgedrängt in die Buhnenfelder, wo nur mit kleinen Geräten, wie Reusen und Körben, gefischt werden kann. Immerhin wird durch die große Zahl dieser Geräte und den hohen Wert des Aales ein beachtlicher Gesamtfang erzielt, der wieder steigende Tendenz zeigt.

Die wichtigsten Nutzfische in der Unterweser sind heute die Wanderfische Aal (Anguilla anguilla L.) und Stint (Osmerus eperlanus L.); die Anlandungen zeigen Tabellen 1 bis 3 .

In der Außenweser spielt die Fischerei auf Krabben, richtiger Garnelen (Crangon crangon L.), eine bedeutende Rolle. Die Anlandungen der 56 Kutter (Abb. 2) erfolgen meist in den kleinen Sielhäfen beiderseits der Wesermündung (Tab. 2).

Der Gesamtwert der Küstenfischerei in der Unter- und Außenweser kann also für 1966 auf fast 3 Millionen DM beziffert werden.

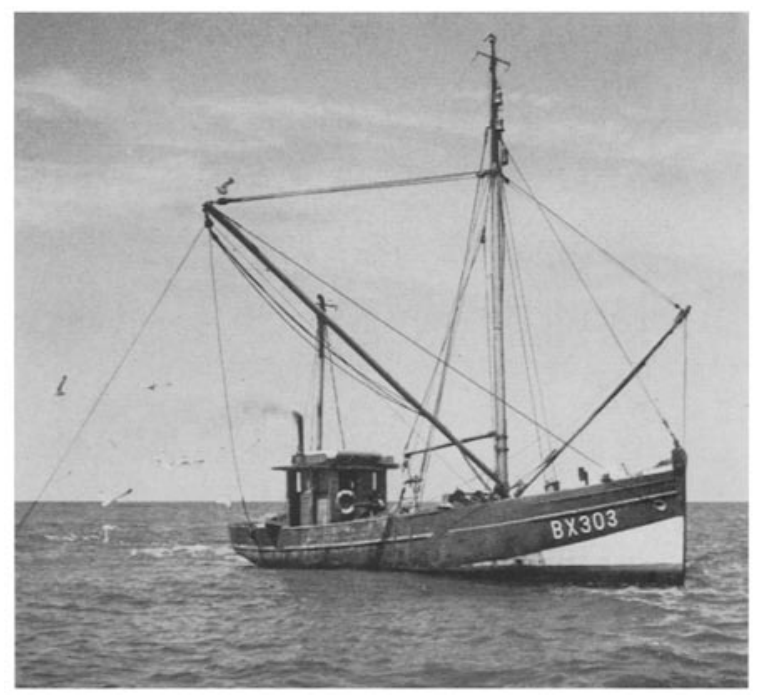

Abb. 2: Krabbenkutter aus Bremerhaven beim Fang mit 2 Schleppnetzen

\section{DIE ABWASSERBEDROHUNG}

Bei dieser Sachlage kann es weder den unmittelbar Betroffenen noch der Offentlichkeit gleichgültig sein, ob die Weser und damit die Fischerei durch Abwässer beeinträchtigt werden. Die bereits vorhandene Abwasserbelastung ist nicht gerade gering. Es ist bekannt, daß die Weser schon oberhalb Bremen eine Versalzung aus der Werra mitbringt. Anliegerstädte wie Minden und Hannover (über Leine und Aller) sorgen für Eutrophierung. Bremen (ca. $200000 \mathrm{cbm}$ Abwässer täglich), Delmenhorst und Oldenburg (über die Hunte) tragen zusätzlich organische Verschmutzungen bei.

Dementsprechend weisen Hustedt (1959) anhand der Diatomeenflora (Formen 
wie Cyclotella, Melosira, Navicula, Nitzschia) und BeHre (1961) auf Grund der Algenbesiedelung (Oscillatoria curviceps, Phormidium autumnale, Cladotrix dichotoma u. a.) die Unterweser insgesamt in das $\alpha$-bis $\beta$-Mesosaprobium ein. Untersucher der Fauna stützen mit ihren Befunden diese Feststellung. SCHRÄDER (1932) beobachtete in der Bodenbesiedlung unterhalb $\mathrm{km} \mathrm{12,5}$ bei Bremen nur noch wenige Insekten und Krebse (Cloeon, Gammarus, Asellus). Als selten bezeichnet er die Weichtiere Pisidium, Valvata, Planorbis und Bythinia. Dagegen fand er viele Oligochaeten wie Stylaria lacustris und insbesondere Tubifex, den typischsten Schmutzbewohner, bei Brake mit 18000 bis 32000 Stück je qm und bei Bremerhaven bis zu 52000 je qm.

FRIEDRICH (1960) stellte heraus, daß unter den Cladoceren die nach LiebmanN (1951) $\beta$-mesosaproben Chydorus sphaericus und Daphnia pulex häufig sind. Sie zeigen in Gegenwart von einigen Oligosaprobien nach seiner Auffassung im ganzen gesehen keinen besonders gefährdeten Zustand. Stärker verschmutzte Lokalstellen sind dabei allerdings nicht in Betracht gezogen worden.

$\mathrm{Zu}$ diesen überwiegend organischen Abwasserbelastungen treten die Abwässer einer Anzahl von Industriewerken, die bereits an der Unterweser heimisch sind.

Die eigentliche Bedrohung sehe ich aber in dem zunehmenden Trend der Industrie, diese Werke auszubauen oder mit neuen großen Werken an die Küste zu gehen, nicht zuletzt in der Meinung, hier seien die Vorfluter so groß und wasserreich, daß man ohne Umstände und Kosten die anfallenden Abwässer los werden könne, und vermutlich sogar in dem guten Glauben, daß hier keine Schäden entstehen können, selbst wenn es sich um besonders unangenehme $A b$ wässer der chemischen Industrie handelt.

Mit größter Sorge sieht daher die Fischerei unter anderem der Errichtung eines großen Industriewerkes bei Blexen, also an der Grenze zwischen Unter- und Außenweser, entgegen.

Mit der Ansiedlung dieses Werkes könnte der Biotop einschneidend verändert werden, denn die allein dort zu erwartenden Abwassermengen mit einem $\mathrm{pH}$-Wert von 1,8 bis 1,9 mit $1590 \mathrm{~m}^{3}$ je Stunde enthalten unter anderem $1080 \mathrm{~kg} \mathrm{H}_{2} \mathrm{SO}_{4}, 270 \mathrm{~kg}$ $\mathrm{FeSO}_{4}, 220 \mathrm{~kg}\left(\mathrm{NH}_{4}\right)_{2} \mathrm{SO}_{4}$ und $160 \mathrm{~kg} \mathrm{TiOSO}_{4}$. Welche Einflüsse von diesen $38000 \mathrm{~m}^{3}$ Abwässern mit $26 \mathrm{t} \mathrm{H}_{2} \mathrm{SO}_{4}$ und 6,5 $\mathrm{t} \mathrm{FeSO}_{4}$ je Tag ausgehen können, besonders wenn sich zusammenbleibende Abwässerkörper bilden, das ist schwer vorauszusehen.

Neuerdings will ein bereits bestehendes Industriewerk künftig täglich $800 \mathrm{~m}^{3}$ Gipssuspension mit einem Feststoffgehalt von $450 \mathrm{t}$ in die Unterweser einleiten. Andere Werke werden folgen. Der einzelne Fall mag jeweils tragbar erscheinen. Wie aber werden Summierung und Akkumulierung wirken?

Die Techniker versuchen mit Vorausberechnungen nachzuweisen, daß ihr Fall keinen Schaden anrichten kann. Dabei gehen sie aus von den Zahlen der Oberwasserzuführung der Weser, die bei Intschede im Minimum 59,7 $\mathrm{m}^{3}$, im Mittel $312 \mathrm{~m}^{3} / \mathrm{sec}$ beträgt. Abgesehen davon, daß dieses Oberwasser schon nicht rein ist, dürfte nach aller biologischen Erfahrung das Minimum bereits eine Gefahrengrenze sein. Aber auch für den Durchschnitt erscheint keineswegs gesichert, daß die theoretischen Wassermengen voll zur Durchmischung mit den eingeleiteten Abwässern kommen können. Die Querschnittfläche der Weser bei Bremerhaven-Blexen beträgt etwa $11000 \mathrm{~m}^{2}$. Bei einer mehr oder weniger punktförmigen Abwassereinleitung kann wohl kaum auf Mitwirkung des gesamten vorhandenen Wassers bei der Durchmischung gerechnet werden, 
auch nicht bei Annahme der Mitwirkung von Tide, Dichteströmungen, Wind und Schiffsverkehr.

\section{DISKUSSION HYDROGRAPHISCHER UND BIOLOGISCHER FORSCHUNGSERGEBNISSE}

Es gibt eine ganze Reihe von wissenschaftlichen Untersuchungsergebnissen, welche die Annahmen der Techniker fraglich erscheinen lassen. Ohne Anspruch auf Vollständigkeit seien eine Anzahl besonders bezeichnender Beispiele angeführt.

\section{Hydrographische Beobachtungen}

Über die ganze Tide bleibt der Salzgehalt des Bodenwassers in der Unterweser fast überall bis zum Beckumer Siel bei km 51,7 höher als der des Oberflächenwassers (Plate 1951).

Am Phosphatgehalt ist mindestens zeitweise eine Schichtung nachzuweisen, in der nach der Abwassereinleitung bei Mittelsbüren in die Weser das $\mathrm{PO}_{4}$ am Nordostufer entlang nur ein Drittel der im übrigen Wasserkörper enthaltenen Menge beträgt (LüNEBURG 1953, zitiert nach HuSTEDT 1957).

BEHRE (1961) folgert aus diesen und seinen eigenen Beobachtungen, daß die in Schiffahrtskreisen immer noch umstrittene Frage, ob durch den reichen Schiffsverkehr, vor allem durch die Bewegung der Schiffsschrauben, die Schichtung in der Weser zerstört wird, wohl eindeutig mit nein beantwortet ist.

Die Trübungswolke im Brackwasser der Weser tritt bei $150 \mathrm{~m}^{3}$ Oberwasser/sec an einer weit höher gelegenen Stelle auf als bei $300 \mathrm{~m}^{3}$ Oberwasser/sec, und die Saumtrübe kann erst dort in das Fahrwasser hineindringen, wo infolge Ausklingens des salzigen Tiefenwassers der vertikale Dichtesprung nachläßt. In hydrographischen Querschnitten durch die Wesermündung stellt sich heraus, daß die rechte Seite der Mündung bei Wremen-Solthörn die ausgesprochene Brackwasserseite mit 5 bis $15 \%$ Salzgehalt und Sinkstoffen bis $1000 \mathrm{mg} / \mathrm{l}$, die gegenüberliegende linke Seite bei Burhave und Fedderwardersiel die eigentliche Flut- und Seewasserseite mit Salzwerten von $20 \%$ und Sinkstoffen von nur $100 \mathrm{mg} / \mathrm{l}$ darstellt (LüNEBURG 1953, 1955).

Es kommt bei großen Flüssen in der Nähe der Einleitungsstelle von Abwässern nicht sofort zu einer vollständigen Durchmischung mit dem Vorflutwasser, sondern es bildet sich eine Abwasserwolke aus, die längere Zeit erhalten bleibt, und die man in der Form der bekannten Abwasserfahne auch in Tidenflüssen weithin flußabwärts verfolgen kann (MANN 1956).

Wassermassen einer bestimmten Konsistenz verschwinden nicht durch Vermischung, sondern bleiben weithin und lange zusammen. Sogar in der Außenweser fast bis Helgoland findet man das Weserwasser als „einen Wasserkörper für sich “ und darüber hinaus existieren noch drei weitere halin und allgemein hydrochemisch wohldefinierte Wassermassen im Gebiet der Weser- und Elbemündung (LüNEBURG 1963). 


\section{Biologische Beobachtungen}

Die hydrographisch unterscheidbaren Wasserkörper müssen mit großer Wahrscheinlichkeit biologisch verschiedene Wirkungen mit sich bringen. KüHL \& MANN (1953) weisen darauf hin, daß extreme Werte in vertikalen Temperatur- und Salzgehaltsschichtungen, auch auf verschiedenen Fahrwasserseiten, für das biologische Geschehen von Bedeutung sein müssen, gerade für das Vorkommen oder Fehlen von Tierarten oder für die Auslösung von Tierwanderungen. Chemismus und biologisches Geschehen stehen in enger gegenseitiger Wechselbeziehung.

In Vertikalzügen in der Unterweser vor der Lunemündung und beim Beckumer Siel finden sich vereinzelt auch die ersten marinen Formen, die hier mit der Flut in dem salzreicheren Tiefenwasser aufwärts treiben. Die Gesamtheit der farblosen Flagellatenformen und die marinen Arten häufen sich unverkennbar in den tieferen Schichten des Wassers (BEHRE 1961).

Es steht fest, daß im Einmündungsgebiet der Flüsse Weser und Elbe höhere Bakterienzahlen zu finden sind als in der freien Nordsee, weiter aber auch, daß das Bakterienmaximum in unterschiedlichen Tiefen anzutreffen ist und daß auch noch auf landfernen Stationen "Wasserinseln mit stark abweichender Bakterienzahl“ beobachtet werden können (GuNKEL 1962).

Neueste Untersuchungen zeigen, daß von Bremerhaven abwärts in der Wesermündung eine Wasserfahne umrissen werden kann, die (fast $50 \mathrm{~km}$ weit) bis an die Leuchttürme Roter Sand und Alte Weser zu verfolgen ist und die dadurch charakterisiert wird, daß in den Sedimenten ihres Bereiches Abwasserbakterien der Coli-Gruppe nachgewiesen werden können (WEYLAND 1967).

Die Abwässer von Cuxhaven schädigen an bestimmten Stellen die Bohrmuscheln (Teredo navalis L.) ebenso wie andere Tiere (KüHL 1958). Zu denken ist auch an den ständigen Rückgang von Hydrobia ulvae in der Deutschen Bucht seit 1923. ZIEGELMEIER (1963) meint dazu, es sollte eingehend untersucht werden, wie weit das allmähliche Zurückgehen der Hydrobia-Bestände gerade am Zusammentreffen des Elbe- und Weserwassers mit dem Chemismus des Wassers oder anderen hydrographischen Gegebenheiten in Zusammenhang steht.

Die russischen Forscher Golikov \& SCARLato (1967) teilen mit, daß nach ihren Untersuchungen in flachen Litoralgebieten die Biozönosen immer mehr zu kleinflächigen Verbreitungsmustern neigen infolge der intensiven Fluktuationen der physikalischchemischen Verhältnisse. Die Verbreitung der Organismen ist eng gebunden an die Positionen bestimmter Wassermassen.

Uberall lassen sich also verschiedenartige Wasserkörper beobachten, die nicht vollständig durchmischt werden und die merkbare biologische Wirkungen hervorrufen. Welcher Art die möglichen Schäden zum Nachteil der Fischerei sein können, das läßt sich vielleicht aus den folgenden Forschungsergebnissen ermessen.

Die Forschergruppe Gamulin-Brida, Giaccone \& Golubić (1967) berichtet, daß die Verschmutzung des Seewassers ein wichtiger ökologischer Faktor ist und zur Störung des natürlichen Gleichgewichts führt.

Nach FrIEDRICH (1952) ist es sicher, daß abiotische Vorgänge eine Änderung der exocönen und dann mittelbar der endocönen Dynamik herbeiführen können. Wir wis- 
sen, daß zum Beispiel starke Phytoplankton-Blüten Sterblichkeit von Wirbellosen und selbst Fischsterben hervorrufen können. Diese Blüten können ihrerseits durch stark düngende Abwässer bewirkt werden.

Untersuchungen von Drebes (1966) über den Diatomeen-Lebenszyklus bestätigen, daß sich abiotische Umweltfaktoren auf die Teilungsrate der Zellen und auf biologische Vorgänge des Formwechsels auswirken, beispielsweise bewirkt Phosphatmangel Dauerporenbildung.

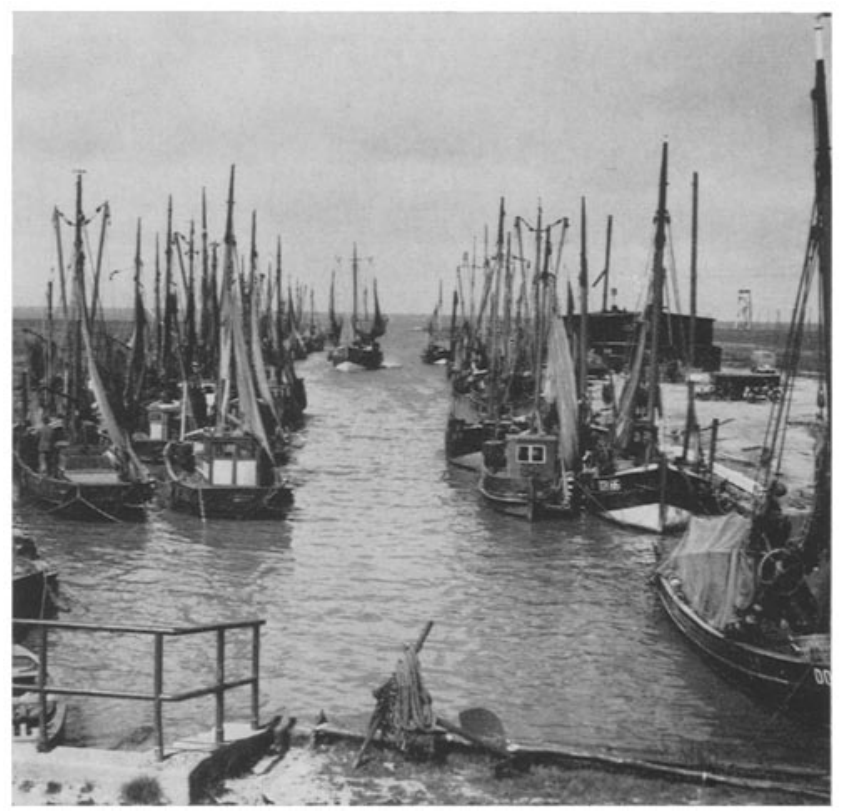

Abb. 3: Typischer Krabbenkutter-Hafen an der Wesermündung (Dorum)

Nach KInNE (1953) können bei physiologisch empfindlicheren Formen der als Fischnahrung besonders wichtigen Gammariden bereits geringe Veränderungen der Biotopverhältnisse zu einem rapiden Produktionsrückgang oder gar zum Absterben großer Bestände führen.

Eine Anderung des $\mathrm{pH}$-Wertes um eine Einheit bewirkt schon eine wesentliche Änderung in der Zusammensetzung der Algenflora (BEHRE 1956). Es scheint möglich, daß bereits ein $\mathrm{pH}$ von unter 6,7 Schäden an den Meeresorganismen hervorruft (ZiJlstra 1966). Nach Zeitzschel (1967) ist die Wasserstoffionenkonzentration als begrenzender Faktor für viele Protozoen wichtig.

Nach Untersuchungen von KRüGER (1966) reagiert der Wattwurm (Arenicola marina) bereits auf minimale Zusätze, zum Beispiel $1 \mathrm{mg} / \mathrm{l}$, Zucker oder Aminosäuren durch Einstellen seiner normalen Pumptätigkeit. Wie wird er auf verdriftete Schwefelsäure reagieren?

Bei mit Fe-Salzen und $\mathrm{H}_{2} \mathrm{SO}_{4}$ angereichertem Abwasser können sich deutliche $\mathrm{Ab}$ - 
lagerungen von Eisenhydroxyd auf den Kiemen von Wassertieren bilden, welche die normale Funktion der Atmung beeinträchtigen (HaLSBAND 1967). Hierher gehören auch die Ergebnisse der Untersuchungen über die Schädigung von Heringseiern und -larven durch Abwässer mit Schwefelsäure und Eisensulfat (KInNE \& RosenthaL 1967).

Für besonders gefährdet halte ich die interstitielle Fauna. Ax (1965) hat in starkem Maße die Aufmerksamkeit auf diese Gruppe gelenkt und auf die Vielfalt der Klassen und Stämme hingewiesen, aus deren Vertretern sich diese Biozönose zusammensetzt wie Cnidarier, Turbellarien, Archianneliden, Polychaeten, Tardigraden, Holoturien und Ascidien. Viele von ihnen gehören sicherlich unmittelbar zur Nahrung der Garnelen und der Jungfische. Neueste Untersuchungen von FLÜCHTER (1966) scheinen das für die junge Seezunge (Solea solea L) zu bestätigen. Der schwedische Biologe GraY (1967) zeigt, daß Archianneliden der interstitiellen Fauna ganz bestimmte Substrate bevorzugen nach Sandkorngröße, bakteriellem Oberflächenfilm und bereits vorhandener Besiedlung, und daß man daher streng lokalisierte Populationen unterscheiden kann. Eine Abwasserfahne kann also wohl nicht ohne Wirkung bleiben.

Organischer Detritus deckt der Sandfauna den Tisch, dagegen verstopfen terrigener Detritus oder zerriebene Kalkmassen das Lückensystem und schädigen die Tiere (REmane 1965). In der Elbe füllt starker Schlickfall vielfach die Sandlücken, so daß dann eine eigentliche interstitielle Fauna gar nicht vorhanden ist (RIEMANN 1966).

Wenn künttig, wie berichtet, ein Werk an der Unterweser täglich $800 \mathrm{~m}^{3}$ Gipssuspensionen mit 450 t Feststoffen einleiten will, so brauchen dort keine Gipsberge auf dem Grunde zu entstehen, es genügt, wenn sich feine Teile unlöslicher Gipsmengen im Sand ablagern, um das Interstitium stark zu verändern und die Fauna dort zu stören oder zu vernichten. Wir wissen nicht, welchen Schaden ein solcher Einbruch in die Nahrungskette verursacht, aber man muß es für sehr unwahrscheinlich halten, daß er ohne jeden Nachteil bleiben würde.

\section{ZUSAMMENFASSUNG}

1. Weder in der Wesermündung noch vor dieser in der Deutschen Bucht kann erwartet werden, daß eingeleitete Abwässer immer "eine schnelle und gute Durchmischung“ oder sogar „eine totale Durchmischung“ erfahren. Es bilden sich sehr häufig Wasserschlieren, Wasserfahnen, Wasserblasen, Wasserkörper oder Wasserinseln, die sich -entgegen allen Theorien und Berechnungen - der totalen Durchmischung entziehen.

2. Die schädlichen Stoffe können mangelhaft oder undurchmischt zusammenbleiben und in mehr oder weniger großen Gebieten biologische Schäden verursachen.

3. Ein Schaden in der biologischen Kette kann weiterwirken auf die Nahrungstiere der Garnelen und Fische und damit mittelbar auf diese selbst oder auf deren Ei- und Jugendstadien, auf den Glasaalaufstieg oder auf Muschelkulturen (KorringA 1967).

4. Es wäre wünschenswert, die Küstengewässer vor großen Abwassereinleitungen zu schützen. Wenn Großsiedlungen und Industrien darauf angewiesen sind, so muß jedenfalls gefordert werden, daß die Technik alle Möglichkeiten ausschöpt, um die Abwässer soweit unschädlich $\mathrm{zu}$ machen, wie es technisch heute möglich ist. Es muß 
erwartet werden, daß Wirtschaftlichkeitsberechnungen nicht nur die Rentabilität der Industrie, sondern auch die Fischerei (Abb. 3) und die Bedeutung der Küste als Erholungsgebiet einbeziehen (CASPERs 1967).

5. Die Fischerei erhebt die über 60 Jahre alten Forderungen für die Binnengewässer (WuNDSCH 1926) heute für die Küstengewässer und das Meer: (a) Das Gewässer darf durch die Abwassereinleitungen nicht so verändert werden, daß die Fischwelt gehindert wird zu leben und sich fortzupflanzen. (b) Die natürliche Fauna und Flora darf nicht vermindert oder in ihrer Zusammensetzung zum Nachteil der Nahrungskette verändert werden. (c) Die technische Durchführbarkeit der Fischerei darf nicht gestört oder unmöglich gemacht werden.

\section{ZITIERTE LITERATUR}

Ax, P., 1966. Die Bedeutung der interstitiellen Sandfauna für allgemeine Probleme der Systematik, Okologie und Biologie. Veröff. Inst. Meeresforsch. Bremerh. (Sonderbd) 2, 15-65.

Berre, K., 1956. Die Algenbesiedlung einiger Seen um Bremen und Bremerhaven. Veröff. Inst. Meeresforsch. Bremerh. 4, 221-384.

- 1961. Die Algenbesiedlung der Unterweser unter Berüdksichtigung ihrer Zuflüsse. Veröff. Inst. Meeresforsch. Bremerh. 7, 71-263.

CAspers, H., 1967. Biologische Gesichtspunkte der marinen Abwasserbelastung. Litoralforschung. In: Bericht über das Kolloquium Abwassereinflüsse in Küstennähe der Dt. ForschGem. in Bremerhaven, 12-15.

Drebes, G., 1966. On the life history of the marine plankton diatom Stephanopyxis palmeriana. Helgolünder wiss. Meeresunters. 13, 101-114.

FlǘCHTER, J., 1966. Spawning, first feeding and larval behavior of the North sea sole. In: C. M. (Council Meeting) I. C.E. S. (International Council for the Exploration of the Sea) Sect. C: Near Northern Seas Committee 3, 1-5.

Friedrich, H., 1952. Ober neuere Gesichtspunkte zur Physiologie der Biocönosen. Veröff. Inst. Meeresforsch. Bremerh. 1, 225-231.

- 1959. Beiträge zu biologisch-ökologischen Untersuchungen der Unter- und Außenweser. Veröff. Inst. Meeresforsch. Bremerh. 6, 1-12.

Gamulin-Brida, H., Giaccone, G. \& Golubrć, St., 1967. Contribution aux études des biocoenoses subtidales. Helgoländer wiss. Meeresunters. 15, 429-444.

Golikov, A. N. \& Scarlato, O. A., 1967. Ecology of bottom biocoenoses in the Possjet Bay (the Sea of Japan) and the peculiarities of their distribution in connection with the physical and chemical conditions of the habitat. Helgoländer wiss. Meeresunters. 15, 193-201.

GRAY, J. S., 1967. Substrate selection by the archiannelid Protodrilus rubropharyngeus. Helgolünder wiss. Meeresunters. 15, 253-269.

GUNKEL, W., 1963. Daten und vorläufige Überlegungen zur Bakterienverteilung in der Nordsee. Veröff. Inst. Meeresforsch. Bremerh. (Sonderbd) 1, 80-89.

HalsBand, E., 1967. Physiologische Untersuchungsmethoden zur Bestimmung des Schädlichkeitsgrades von Abwassergiften im Süß-, Brack- und Salzwasser. Litoralforschung. In: Bericht über das Kolloquium Abrvassereinflïsse in Küstennähe der Dt. ForschGem. in Bremerhaven, 20-22.

Hustedt, F., 1957. Die Diatomeenflora des Flußsystems der Weser im Gebiet der Hansestadt Bremen. Abb. naturw. Ver. Bremen, 34, 181-440.

- 1959. Die Diatomeenflora der Unterweser von der Lesummündung bis Bremerhaven mit Berücksichtigung des Unterlaufes der Hunte und Geeste. Veröff. Inst. Meeresforsch. Bremerh. 6, 13-176.

KInNe, O., 1953. Zur Biologie und Physiologie von Gammarus duebeni LiLlJ. VI. Produktionsbiologische Studie. Veröff. Inst. Meeresforsch. Bremerb. 2, 135-145. 
- \& Rosenthal, H., 1967. Effects of sulfuric water pollutants on fertilization, embryonic development and larvae of the herring, Clupea harengus. Mar. Biol. 1, 65-83.

KorRINGA, P., 1967. Organisation und Biologie der Abwasserbeseitigung im Meer in den Niederlanden. Litoralforschung. In: Bericht über das Kolloquium Abrwassereinflïsse in Küstennähe der Dt. ForschGem. in Bremerhaven. Anh., 1-5.

KRÜGER, F., 1966. Experimentelle Untersuchungen am Wattwurm. Jber. Biol. Anst. Helgoland, 1966, Ca 24-28.

KüHz, H., 1958. Das Auftreten mariner Holzschädlinge (Bohrmuscheln und Bohrkrebse) in Flußmündungen und Häfen in Abhängigkeit von den Wasserverhältnissen. Arb. dt. Fisch. Verb. 10, 19-34.

- \& Mann, H., 1953. Beiträge zur Hydrochemie der Unterelbe. Veröff. Inst. Meeresforsch. Bremerh. 2, 236-268.

LiebManN, H., 1951. Handbuch der Frischwasser- und Abwasserbiologie. R. Oldenbourg, Mündhen 1951, Bd 1, 419.

Lüneburg, H., 1953. Probleme der Sinkstoffverteilung in der Wesermündung. Veröff. Inst. Meeresforsch. Bremerh. 2, 15-51.

- 1955. Die Verteilung der Sinkstoffe in den Seitenräumen der Wesermündung. Veröff. Inst. Meeresforsch. Bremerb. 3, 228-265.

- 1963. Wassermischvorgänge vor der Weser- und Elbmündung. Veröff. Inst. Meeresforsch. Bremerh. 8, 111-141 und Sonderbd 1, 95-100.

ManN, H., 1956. Aufarbeitung von Abwasser in Tideflüssen (Elbe). Arb. dt. Fisch. Verb. 7, $8-16$.

MÜller, K., 1960. Hat der Weserlachs noch eine Zukunf? Weser 34, 19-21.

Plate, H., 1951. Die Salzgehaltverhältnisse im Brackwassergebiet der Unterweser. Nenes Arch. Nieders. 25, 497-516.

Remane, A., 1966. Einfluß von organischem und anorganischen Detritus auf die Sandfauna. Diskussions-Beitr. Veröff. Inst. Meeresforsch. Bremerh. (Sonderbd) 2, 207.

Rremann, F., 1966. Die Verbreitung der interstitiellen Fauna im Elbe-Astuar. Veröff. Inst. Meeresforsch. Bremerh. (Sonderbd) 2, 117-123.

SCHRÄDER, T., 1932. Fischereibiologische Untersuchungen im Wesergebiet. Z. Fisch. 30, 648-680.

WeYLAND, H., 1967. Uber die Verbreitung von Abwasserbakterien im Sediment des Weserästuars, Veröff. Inst. Meeresforsch. Bremerh. 10, 173-182.

Wundsch, H. H., 1926. Die Reinhaltung unserer Fischgewässer. In: Handbuch der Binnenfischerei Mitteleuropas. Hrsg. von H. H. Wundsch. Schweizerbart, Stuttgart, Bd 6, 140-221.

Zeitzschel, B., 1967. Die Bedeutung der Tintinnen als Glied der Nahrungskette. Helgoländer wiss. Meeresunters. 15, 589-601.

Ziegelmeier, E., 1963. Das Makrobenthos im Ostteil der Deutschen Bucht. Veröff. Inst. Meeresforsch. Bremerh. (Sonderbd) 1, 101-114.

ZijLstra, K. C., 1966. Welke stoffen komen in aanmerking voor lozing in zee? Water $\mathbf{5 0} 417$.

\section{Diskussion im Anschluß an den Vortrag Norte}

WEBER: Für eine sichere Vorhersage über die Schäden durch Einleitung von gereinigten Abwässern in Flußmündungen und in das Küstenmeer reichen die heute bekannten Unterlagen nicht aus. Forschungen auf diesem Sektor sind daher sehr zu begrüßen. Durch geeignete Ausgestaltung des Einleitungsbauwerks kann eine schlechte Verteilung des Abwassers im Fluß vermieden werden. Die in die Unterweser einzubringenden Gipskriställchen werden sich zum großen Teil auflösen, da im Wasser noch ein Lösungsvermögen für Gips vorhanden sein dürfte. Ein Sedimentieren des Gipses wird daher nur in geringem Umfange zu erwarten sein.

LüNeBurg: Seit Jahren verklappt die Titan-Gesellschaft westlich von Rotterdam die umstrittene „Dünnsäure“. Welche Erfahrungen haben die holländischen Kollegen bezüglich der Sedimentbeeinflussung durch z. B. kolloides $\mathrm{Fe}(\mathrm{OH})_{3}$ (Limonite) gemacht? 
KoRRINGA: We have experienced that sulfuric acid itself, if it is properly distributed, not in one site but by a boat moving in a well-defined area, is very easily taken up by the well buff-ered seawater without leading to any noticeable changes. Moreover we have made some observations in that area on the bottom. As far as this area west of Rotterdam we have not seen much damage. In accordance with what Dr. WeBER has said, I think it will be very important if countries around the North Sea select certain stations where they take bottom samples with a certain frequency, once a month or once a quarter, and to analyse very carefully in this bottom sample the interstitial fauna to see whether there are gradual changes and whether perhaps certain species disappear, and then one should not look for very rare species, but for those which really play a part in the food cycle. Thus we can observe whether there are any changes. We should not be too late in doing this to see if there are any gradual changes which might be due to waste disposal.

NoLTE: Es ist richtig, daß wir vieles noch nicht wissen, daß also die Betroffenen noch nicht mit fertigen Vorschriften versehen werden können. Es wird sich aber wohl niemand dem Eindruck entziehen können, daß intensiy an den Problemen gearbeitet wird, und daß wir demnächst bessere Kenntnisse haben werden. Das darf uns aber nicht veranlassen, zu großzügig zu werden. Ein warnendes Beispiel ist Japan. Dort spielt die Fischerei eine große Rolle. Aber auch die Industrie macht große Anstrengungen, auf dem Weltmarkt konkurrenzfähig zu sein. Dort war man offenbar zu großzügig. Die Folge ist, daß die durch Abwasserverschmutzung verursachten Schäden in der Küstenfischerei bereits auf 12 bis 13 Milliarden Yen beziffert werden, oder nach unserer Rechnung etwa 150000 Millionen DM. Das sollte uns zur Vorsicht mahnen. 